\title{
Primary schwannoma of the diaphragm
}

\author{
Eliana Abraham ${ }^{\mathrm{a}}$, and Gary Schwartz, MD ${ }^{\mathrm{b}}$

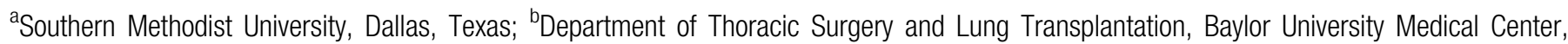 \\ Dallas, Texas
}

\section{ABSTRACT}

Primary schwannoma of the diaphragm is exceedingly rare. We report a case identified on surveillance imaging for a previous lung cancer that was diagnosed via thoracoscopic resection.

KEYWORDS Diaphragm mass; schwannoma; video-assisted thoracic surgery

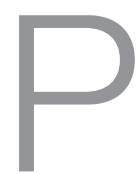

rimary tumors of the diaphragm are uncommon, with most benign lesions being cystic or lipomatous and the most common malignant etiology being rhabdomyosarcoma. ${ }^{1,2}$ Treatment is typically observation unless the tumor is symptomatic or the diagnosis is unknown with concern for malignancy. Primary schwannoma of the diaphragm is exceedingly rare, with less than 10 cases reported to date; three cases were reported in the pediatric population and one case was reported of a malignant schwannoma. ${ }^{3-5}$ We report a case of primary schwannoma of the diaphragm identified on surveillance imaging for a history of lung cancer and diagnosed after resection via a thoracoscopic approach.

\section{CASE DESCRIPTION}

A 68-year-old female former smoker presented with a chronic cough and was found to have a medial right lower lobe infiltrate and a left lower lobe solid nodule. The left lower lobe nodule remained stable during surveillance; however, the right lower lobe lesion progressed. The patient underwent surgical exploration and was found to have lepidic growth adenocarcinoma, for which a right lower lobectomy and right upper lobe posterior segmentectomy were performed. She recovered well from surgery, but on surveillance imaging 6 months later the left lower lobe nodule had enlarged (Figure 1). Although it appeared radiographically benign, it was not amenable to percutaneous biopsy.
Given her previous history of cancer, she elected for surgical resection.

She underwent left video-assisted thoracic surgery with a plan for wedge resection of the presumed pulmonary nodule. Upon exploration of the chest, the mass was noted to be approximately $2 \mathrm{~cm}$ and arising from the body of the diaphragm with no invasion into the left lower lobe (Figure 2). It was resected full thickness with a wide margin using electrocautery, and the diaphragm was reconstructed primarily using an interrupted 3-0 Prolene suture. She was discharged home on the same day and had an uneventful recovery. Final pathological analysis revealed a benign schwannoma positive for $S 100$ and CD34 immunohistochemical stains. Serial imaging 1 year after cancer resection demonstrated no evidence of recurrent disease.

\section{DISCUSSION}

Schwannomas are tumors of the nerve sheath that can occur anywhere in the peripheral nervous system and are typically slow growing. Although they are typically not cancerous, malignant cases have been reported and even benign lesions can be symptomatic due to nerve damage as well as compression of adjacent structures.

This case highlights the variety of anatomic locations where these tumors can occur, as well as their predilection to mimic more aggressive tumors. Most cases of benign diaphragmatic masses can be observed with serial imaging and only require resection if the diagnosis is unknown or if compressive symptoms develop. In this case the concern for

Corresponding author: Gary Schwartz, MD, Department of Thoracic Surgery and Lung Transplantation, Baylor University Medical Center, 3410 Worth St., Suite 545, Dallas, TX 75246 (e-mail: Gary.Schwartz@BSWHealth.org)

Color versions of one or more of the figures in the article can be found online at www.tandfonline.com/ubmc.

Received September 4, 2018; Revised November 20, 2018; Accepted November 26, 2018. 


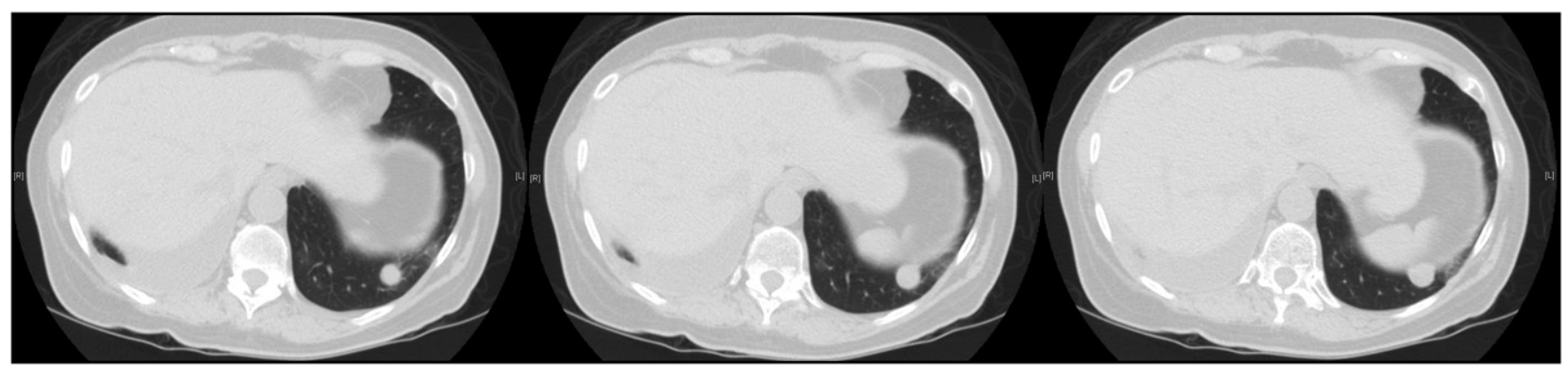

Figure 1. Computed tomography scan of the chest demonstrating a left pleural-based mass that appears to be intraparenchymal.

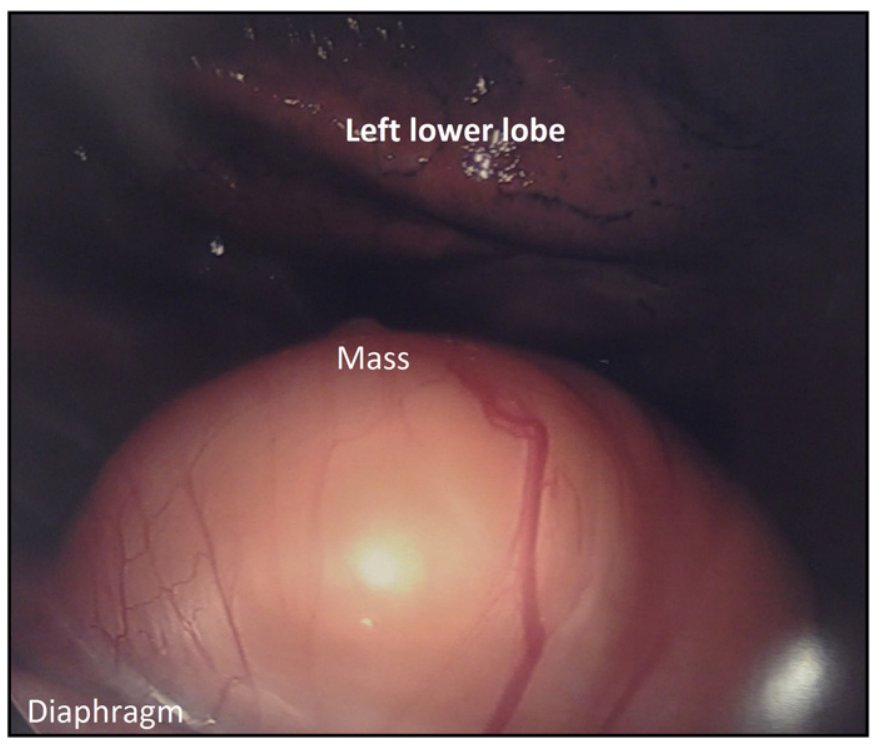

metastatic lung cancer prompted resection, which was performed in a minimally invasive fashion.

1. Wiener MF, Chou WH. Primary tumors of the diaphragm. Arch Surg. 1965;90(1):143-152. doi:10.1001/archsurg.1965. 01320070145031.

2. Kim MP, Hofstetter WL. Tumors of the diaphragm. Thorac Surg Clin. 2009;19(4):521-529. doi:10.1016/j.thorsurg.2009.08.007.

3. Kumbasar U, Enon S, Osman Tokat A, Gungor A. An uncommon tumor of the diaphragm malignant schwannoma. Interact Cardiovasc Thorac Surg. 2004;3(2):384-385. doi:10.1016/j.icvts. 2004.01.021.

4. Ohba T, Shoji F, Kometani T, et al. Schwannoma in the peridiaphragm. Gen Thorac Cardiovasc Surg. 2008;56(9):453-455. doi:10.1007/s11748-008-0269-0.

5. Hobbs DJ, McLellan J, Schlatter MG. Schwannoma of the diaphragm - a pediatric case report and review of the literature. $J$ Pediatr Surg. 2012;47(4):e5-e7. doi:10.1016/j.jpedsurg.2011.11.065.

Figure 2. Thoracoscopic view of the left pleural space. The mass arises from the diaphragm without invasion into the left lower lobe. 